\title{
Sensitivity Jump of Micro Accelerometer Induced by Micro- fabrication Defects of Micro Folded Beams
}

\author{
Wu Zhou ${ }^{1}$, Lili Chen ${ }^{2}$, Huijun $\mathrm{Yu}^{1}$, Bei Peng ${ }^{1}$, and $\mathrm{Yu}$ Chen ${ }^{1}$ \\ ${ }^{1}$ School of Mechatronics Engineering, University of Electronic Technology and Science of China, 2006 Xiyuan Ave., \\ 611731, Chengdu, China, zhouwu916@uestc.edu.cn \\ ${ }^{2}$ College of Mechanical Engineering, Chengdu Technological University, 1 Zhongxing Ave., 611730, Chengdu, China
}

\begin{abstract}
The abnormal phenomenon occurring in sensor calibration is an obstacle to product development but a useful guideline to product improvement. The sensitivity jump of micro accelerometers in the calibrating process is recognized as an important abnormal behavior and investigated in this paper. The characteristics of jumping output in the centrifuge test are theoretically and experimentally analyzed and their underlying mechanism is found to be related to the varied stiffness of supporting beam induced by the convex defect on it. The convex defect is normally formed by the lithography deviation and/or etching error and can result in a jumping stiffness of folded microbeams and further influence the sensitivity when a part of the bending beams is stopped from moving by two surfaces contacting. The jumping level depends on the location of convex and has nothing to do with the contacting properties of beam and defects. Then the location of defect is predicted by theoretical model and simulation and verified by the observation of micro structures under microscopy. The results indicate that the tested micro accelerometer has its defect on the beam with a distance of about $290 \mu \mathrm{m}$ from the border of proof mass block.
\end{abstract}

Keywords: MEMS, micro accelerometer, stiffness, sensitivity, defect.

\section{INTRODUCTION}

Micro accelerometers need to be calibrated precisely before being introduced into markets or applications [1], [2]. Any abnormal phenomena occurring in the calibrating process must be identified by either a direct observation or an indirect evaluation to acquire guidelines for a more mature design [3]. It is one thing to find such abnormal behavior, quite another to model the underlying physical mechanism because different devices involve different mechanisms even under the same abnormalities. A certain abnormality which even occurs only once in hundreds of or thousands of tests always draws intense attention of researchers to search for a rational and reasonable explanation to it. The searching processes and results are recognized as a practical case study which is utilized to find out the optimization direction of a design or to test a new methodology or to extend a renewed application [4]-[8].

Micro accelerometer, as a crucial part of inertial navigation systems, has been intensively studied since the 1980s. The ever-growing fabrication and IC technology are driving micro accelerometers to the high precise application fields where higher stability and reliability are specifically required [9], [10]. Therefore, any uncertainties during design, fabrication, packaging and calibration of accelerometer cannot be neglected. Due to the uniqueness of failure mode in micro device, the accelerometer studied in this paper is a glass-on-silicon comb structure fabricated by bulk machining process and anodic bonding. The details can be found in authors' other publications [11]-[13]. Fig.1. gives the diagram and SEM (Scanning Electron Microscope) of comb capacitive accelerometer whose output is the differential capacitance between two separate groups of comb capacitors. Each capacitor unit consists of two parallel plate electrodes. One is fixed finger mounted to the substrate and the other is movable finger attached to the mass. The gap between the fixed and moveable fingers can be changed by the movement of mass resulting from the inertial force formed by input acceleration. The capacitance, according to the electric theory, then varies with the changed gaps to form a changed differential signal which is measured by an external circuit to provide an electrical output such as a DC voltage [12], [14], [15]. The output voltage of external circuit under an ideal condition is proportional to the input acceleration along the sensitive axis of micro accelerometers.

What this paper concerns is the sensitivity jump during the calibration of micro accelerometer on centrifuge. In section 2 , the calibration process is given in details to show what the sensitivity jump is. Section 3 suggests the cause of such an unusual behavior is related to the stiffness jump of sensors, followed by a complete analysis of the test data and a microscopy observation of micro structures. And the conclusion is given in Section 4 . 


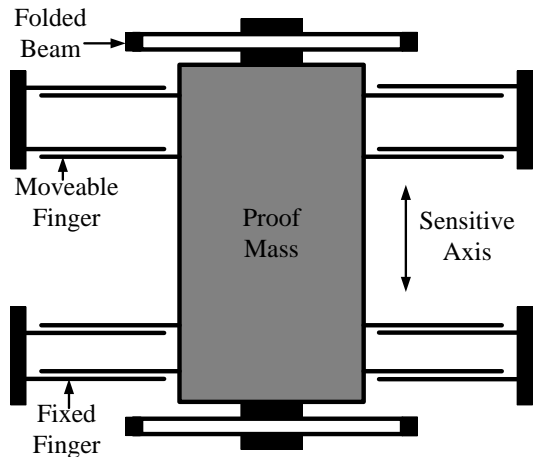

a) Diagram of sensor

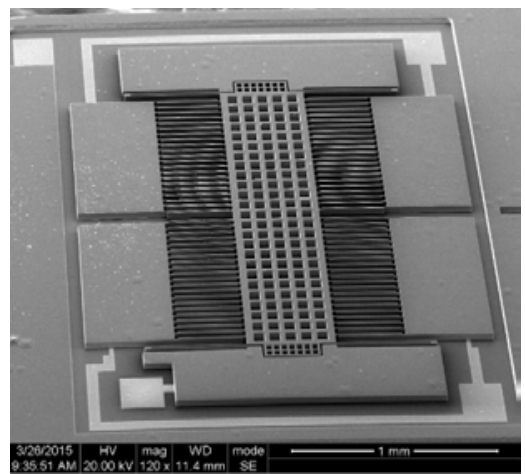

b) SEM of accelerometer

Fig.1. Typical structure of comb accelerometer.

\section{CALIBRATION METHOD}

In calibration procedure, the sensitive axis of micro accelerometer is set along with the radial direction of the circumrotating platform. Thus, the input acceleration is equal to the centripetal acceleration resulting from uniform circular motion of the centrifuge platform. The acceleration range is from $-30 g_{0}$ (where $g_{0}$ is the standard acceleration due to gravity) to $30 g_{0}$ with the increment of $5 g_{0}$. The full range calibration is divided into two steps as $0 \sim 30 g_{0}$ and 0 $\sim-30 g_{0}$ due to unidirectional acceleration provided by centrifuge. The output of accelerometer is represented by DC voltage recorded by data acquisition system. The acquired value of voltage at each calibration point is an average value of 20 records under a stable state. To avoid data singularities, each direction test includes an increasing step, $0 \rightarrow 30 g_{0}$, and a decreasing step, $30 g_{0} \rightarrow 0$, with increments of $5 g_{0}$ and $-5 g_{0}$, respectively. The test data obtained from an abnormal accelerometer are listed in Table 1. Once being shown as the red line and marks in Fig.2., the test data exhibits an abnormal phenomenon as the input acceleration exceeds $15 g_{0}$ in the positive direction by comparing with the normal ones marked by blue lines and diamonds.

It is concluded from Table 1. and Fig.2. that the accelerometer can work normally in the range of $-30 g_{0} \sim$ $+15 g_{0}$ and shows a deviation in the range of $+20 g_{0} \sim+30 g_{0}$. And the deviated data keeps a good linear relationship between input and output data with a changed slope compared with the normal range.

\section{ANALYSIS \& DERIVATION}

According to the working principle of micro accelerometers, what relates the output voltage to the input acceleration is the differential capacitance of comb capacitors constructed by fixed plates on substrate and movable plates on mass. The relationship can be simply expressed as:

$$
V_{o}=\frac{M\left(C_{1}-C_{2}\right)}{C_{1}+C_{2}} \cdot f(T)
$$

where $V_{o}$ is the output voltage; $M$ is the amplification factor of sensing circuit; $C_{1}$ and $C_{2}$ are the capacitances formed by two separated capacitor groups on sensors, respectively; $f(T)$ is a function relating to working temperature at which the data are acquired.

Table 1. Test results of some abnormal accelerometer.

\begin{tabular}{cccc}
\hline \multicolumn{2}{c}{ Positive direction } & \multicolumn{2}{c}{ Negative direction } \\
Input $\left[g_{0}\right]$ & Output[V] & Input $\left[g_{0}\right]$ & Output[V] \\
\hline 0 & -0.251064 & 0 & -0.252078 \\
5 & 0.444109 & -5 & -0.947177 \\
10 & 1.151835 & -10 & -1.652348 \\
15 & 1.882528 & -15 & -2.376899 \\
20 & 2.232179 & -20 & -3.132769 \\
25 & 2.749463 & -25 & -3.931570 \\
30 & 3.266746 & -30 & -4.784545 \\
25 & 2.749301 & -25 & -3.932403 \\
20 & 2.231855 & -20 & -3.133117 \\
15 & 1.883392 & -15 & -2.377323 \\
10 & 1.152557 & -10 & -1.650463 \\
5 & 0.444413 & -5 & -0.947249 \\
0 & -0.252078 & 0 & -0.252249 \\
\hline
\end{tabular}

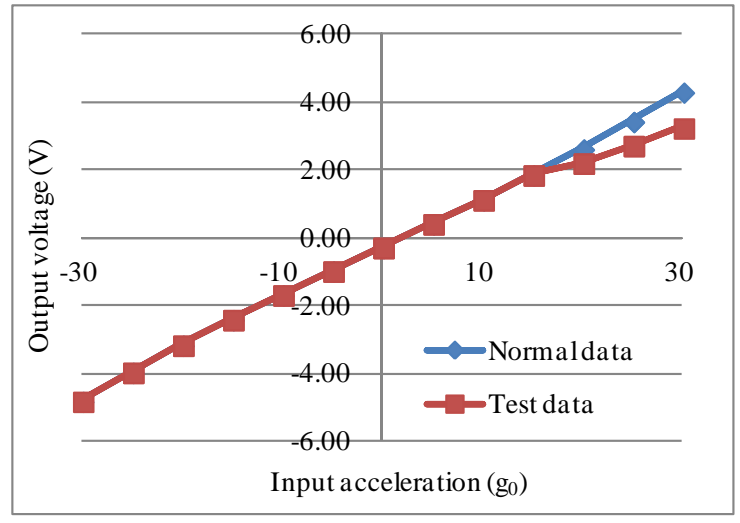

Fig.2. Test data on centrifuge vs. should-be data.

During the test process, the amplification factor $M$ is a constant and the temperature function $f(T)$ keeps invariant or its variation is small enough to be ignored due to the constant temperature environment of centrifuge. The 
capacitance is formed by sensing capacitors, therefore, is the crucial element to inducing the sharp change of output voltage. The capacitance is determined by three factors: permittivity of dielectric, overlap area of plates and gaps of parallel plates. During these factors, the permittivity stays constant because of the hermetical packaging and the overlapped area has almost no change in the test because the inertial force generated by acceleration is vertical to plates [11]. Hence the capacitor gap which is a sensitive physical parameter in capacitance sensing schemes is identified as the only key factor to the sensitivity jump. The gap change is related to the movement of proof mass block formed by external acceleration. And for the micro accelerometers, the level of gap change depends on both the amplitude of acceleration and the stiffness of supporting beams. According to the operating principle of accelerometer, the relationship between output and input can be linearly expressed as:

$$
V_{o}=K_{0}+K_{1} \cdot \Delta g=K_{0}+\frac{K_{1} m a}{K_{b}}
$$

where $K_{0}$ is the zero offset of sensor, its unit is volt, $\mathrm{V} ; K_{1}$ is a constant determined by structural dimensions and circuit parameters, its unit is volt per meter, $\mathrm{V} / \mathrm{m} ; m$ is the mass of proof mass, its unit is kilogram, kg; $a$ is the input acceleration with unit as $\mathrm{m} / \mathrm{s}^{2}$; and $K_{b}$ is the stiffness of supporting beams with unit as $\mathrm{N} / \mathrm{m}$.

According to (2), the voltage and acceleration keep a good and identical linear relationship in the whole measurement range [12], which is the substantial requirement of micro accelerometer design. The abnormal phenomenon, however, occurs when the acceleration increases to a certain point (Fig.2.) and another linear relationship emerges (Fig.3.). The different slopes appear between normal data and test data in the range of $+20 g_{0} \sim+30 g_{0}$; it means the sensitivity, defined by the ratio of output voltage and input acceleration, $\mathrm{V} / \mathrm{g}_{0}$ or $\mathrm{mV} / \mathrm{g}_{0}$, jumps from one value to another. Such an unusual behavior is called sensitivity jump in this paper. The sensitivity of the tested accelerometer shifts from $166.9 \mathrm{mV} / g_{0}$ to $103.5 \mathrm{mV} / g_{0}$ once acceleration exceeds $20 g_{0}$ in positive direction but keeps identical in negative direction. Besides, the increase of output voltage with increased acceleration means the movability of proof mass is not stopped but decreased. It indicates that parameter $K_{b}$ in (2) experiences some changes during the test process because other parameters are constants.

The reduction of sensitivity must result from an increase of stiffness constant of beams which is a folded structure constructed by four fixed-fixed beams in parallel [16], [17]. A stiffness shift cannot be generated by expanding or shrinking the structural dimensions of a fabricated beam but can be induced by the deformation of beams, such nonlinearity, sticking or defects [6], [18], [19]. However, the nonlinearity is not the contributor to the mentioned abnormal behavior because the changed slope exhibits a good linearity and the sticking can be easily excluded by the movability of mass block in both direction and the return of stiffness to normal state after decreasing acceleration. The defects, therefore, are the one and only factor left to giving rise to the sensitivity jump.

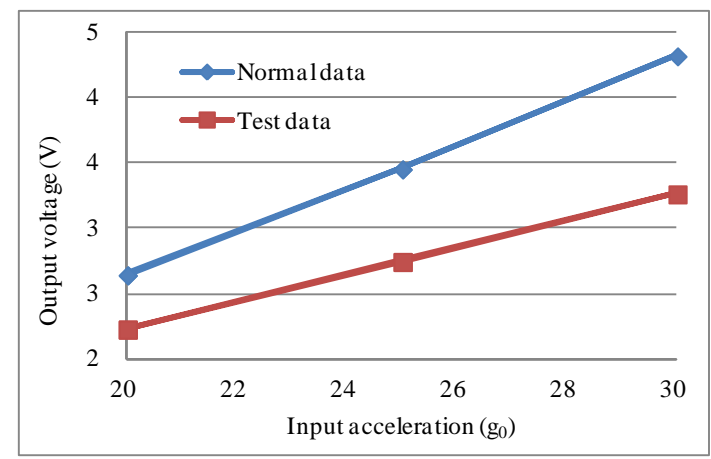

Fig.3. Sensitivity jump shown by different slopes.

The defects in micro structures are mainly caused by the micro manufacturing process, for example, the dry/wetetching induces sidewall roughness or angled grooves, the over-etching results in concave defects and under-etching leads to convex defects [20]-[24]. Both the roughness and concaves impact the stiffness of micro beams only in the form of nonlinearity, while the convex on beam will change the effective stiffness by stopping the movement of a certain segment of beam when the deformation forms a contact between two adjacent parts. As illustrated in Fig.4., the cantilever undergoes a bending deflection under an applied force, F, at its free end. The stiffness before beam (segment A) contacting convex is much different from that after contacting because the cantilever transforms into a compound beam consisting of two separated beams in series. This difference induced by the obstacle can generate a stiffness jump and then can cause a sensitivity shift of micro devices.

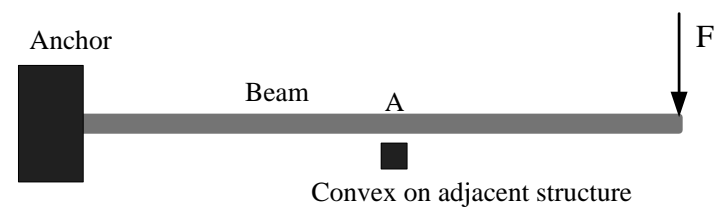

Fig.4. Effective stiffness is changed by convex due to contact.

A disassembly process of the packaged accelerometer is carried out to investigate, in microscopy by continuously adjusting its focus plane, whether there are any convex defects existing in the sensor structure. The observation in Fig.5.a) indicates a convex appearing between two parallel beams of folded beam in Fig.5.b). A simplified model of folded beam is described in Fig.5.c) where the convex defect is represented by a rectangle segment.

The following contents in this section are to derive the stiffness constant of beam with a convex defect (Fig.5.) to evaluate the influences. The folded beam is constructed by combining two flexible beams in series through a rigid rectangle joint. Therefore, it can be divided into two main parts - the upper beam of Fig.6.b) and the lower beam of 
Fig.6.c). The coordinates and directions of forces and moments acting on beams are defined in Fig.6. Only the contacting state is concerned in following calculations because the noncontacting state can be easily obtained in other publications [16], [18], [19].

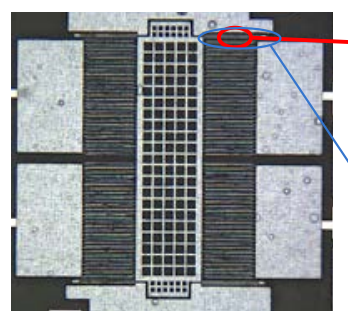

(a) Sensor structure

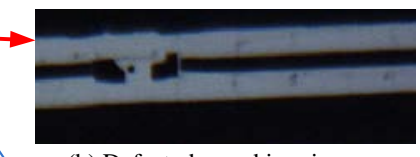

(b) Defect obseved in microscopy Anchor
Mass

(c) Half structure of folded beam with defect
Fig.5. Convex on folded beam.

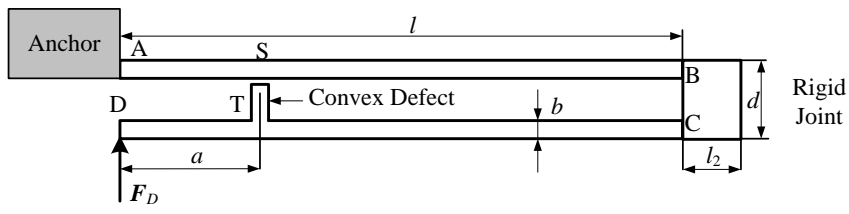

(a) Folded beam with defect

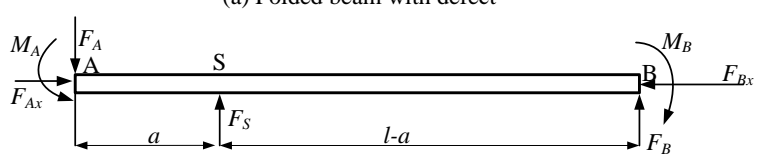

(b) The top beam

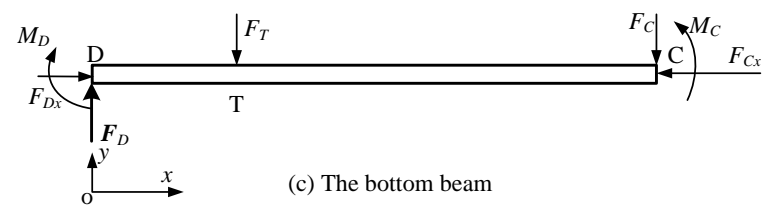

Fig.6. Folded beam with convex defect.

The equations of bending moments of upper beam, neglecting the friction of the contacting surface, can be divided into two segments by the contacting point, $\mathrm{S}$, and expressed as:

$$
\begin{gathered}
M(x)=-\left(M_{A}+F_{A} x\right),(0<x \leq a) \\
M(x)=-M_{A}-F_{A} a-F_{B}(x-a),(a<x<l)
\end{gathered}
$$

where $M(x)$ represents the bending moment of the section at distance $x$ from the left end, $M_{A}$ and $F_{A}$ represent the moment and force acting on the beam from the anchor, respectively. $F_{B}$ represents the force acting on the beam from the joint.

According to beam bending theory [25], the differential equation of beam deflection is expressed as:

$$
E I_{z} w^{\prime \prime}=M(x)
$$

where $E$ is the Young's modulus; $I_{z}=h b^{3} / 12$ is the second moment of the cross-sectional area, $h$ is the thickness of the beam and $b$ is the width of the beam; $w$ is the deflection.
According to the geometric deformation of beams, the boundary conditions of the differential equation are that both the slope, $w^{\prime}$, and displacement, $w$, at $x=0$ are equal to zero.

Substitute the bending moment into the deflection equation, the deflection and slope of each segment can be expressed as:

$$
\begin{gathered}
E I_{z} w^{\prime}=-\frac{F_{A}}{2} x^{2}-M_{A} x,(0<x \leq a) \\
E I_{z} w=-\frac{F_{A}}{6} x^{3}-\frac{M_{A}}{2} x^{2},(0<x \leq a) \\
E I_{z} w^{\prime}=-\frac{F_{B}}{2} x^{2}-\left(M_{A}+F_{A} a-F_{B} a\right) x \\
+\frac{F_{A} a^{2}}{2}-\frac{F_{B} a^{2}}{2},(a<x<l) \\
E I_{z} w=-\frac{F_{B}}{6} x^{3}-\frac{M{ }_{A}+F_{A} a-F_{B} a}{2} x^{2}+ \\
\frac{F_{A}-F_{B}}{2} a^{2} x+\frac{F_{B} a^{3}}{6}-\frac{F_{A} a^{3}}{6},(a<x<l)
\end{gathered}
$$

As for the lower beam, the process of deriving is similar to the upper one. The deflection equations can be expressed as:

$$
E I_{z} w=\frac{F_{D}}{6} x^{3}+\frac{M_{D}}{2} x^{2}+D_{1}
$$

$$
E I_{z} w=\frac{1}{6} F_{C} x^{3}+\left(\frac{M_{D}}{2}+\frac{F_{D} a}{2}-\frac{F_{C} a}{2}\right) x^{2}+D_{2} x+D_{3}
$$

with the boundary conditions as:

$$
\begin{gathered}
w^{\prime}(0)=0 \\
w^{\prime}(a)=\left(M_{D} a+\frac{F_{D} a^{2}}{2}\right) /\left(E I_{z}\right) \\
w^{\prime}(l)=\frac{2\left(-M_{A} l-F_{A} a l+F_{B} a l\right)-F_{B} l^{2}+F_{A} a^{2}-F_{B} a^{2}}{2 E I_{z}} \\
w(a)=\left(\frac{M_{A} a^{2}}{2}+\frac{F_{A} a^{3}}{6}+D_{3}\right) /\left(E I_{z}\right) \\
+\frac{-6 M_{A} l^{2}-6 F_{A} a l^{2}+6 F_{B} a l^{2}-F_{B} l^{3}}{6 E I_{z}} \\
6 E I_{z} a^{2} l-3 F_{B} a^{2} l+F_{B} a^{3}-F_{A} a^{3}
\end{gathered}
$$


where $M_{D}$ and $F_{D}$ represent the moment and force acting on the beam from the proof mass, respectively. And the variables in equations can be expressed as:

$$
\begin{aligned}
& D_{1}=-M_{A} l^{2}-F_{A} a l^{2}+F_{B} a l^{2}-\frac{F_{B} l^{3}}{3} \\
&+ F_{A} a^{2} l-F_{B} a^{2} l+\frac{F_{B} a^{3}}{3}-\frac{F_{A} a^{3}}{3} \\
& D_{2}=\frac{F_{C} a^{2}}{2}-\frac{F_{D} a^{2}}{2} \\
& D_{3}=-M_{A} l^{2}-F_{A} a l^{2}+F_{B} a l^{2}-\frac{F_{B} l^{3}}{3} \\
&+F_{A} a^{2} l-F_{B} a^{2} l+\frac{F_{B} a^{3}}{6}-\frac{F_{A} a^{3}}{6}
\end{aligned}
$$

Additionally, the state of static equilibrium can lead to $M_{A}=M_{D}, F_{A}=F_{D}$ and $F_{B}=F_{C}$. Hence the deflection of left end $\mathrm{D}$ under an external force $F_{D}$ can be expressed as:

$$
\begin{aligned}
w_{D}= & \frac{-M_{A} l^{2}}{E I_{z}}+\left(a l^{2}-\frac{l^{3}}{3}-a^{2} l+\frac{a^{3}}{3}\right) \frac{F_{B}}{E I_{z}} \\
& +\left(a^{2} l-a l^{2}-\frac{a^{3}}{3}\right) \frac{F_{D}}{E I_{z}}
\end{aligned}
$$

which has

$$
\begin{aligned}
& M_{\mathrm{A}}=-\frac{F_{\mathrm{D}}}{l}\left[a l-\frac{1}{2} a^{2}+\right. \\
&\left.\frac{a l-\frac{1}{2} a^{2}-\frac{a l^{2}}{a^{2}-l^{2}}\left(-a l+\frac{1}{2} l^{2}+\frac{1}{2} a^{2}\right)}{a l-\frac{1}{2} l^{2}-\frac{1}{2} a^{2}+\frac{l}{a^{2}-l^{2}}\left(a l^{2}-\frac{1}{3} l^{3}-a^{2} l+\frac{1}{3} a^{3}\right)}\right] \\
& F_{B}=F_{\mathrm{D}} \frac{a l-\frac{1}{2} a^{2}-\frac{a l^{2}}{a+l}}{a l-\frac{1}{2} l^{2}-\frac{1}{2} a^{2}+\frac{l}{a^{2}-l^{2}}\left(a l^{2}-\frac{1}{3} l^{3}-a l^{2}+\frac{1}{3} a^{3}\right)}
\end{aligned}
$$

The accelerometer sensor, symmetrically designed to guarantee the movement of end $\mathrm{D}$ of folded beam, is parallel with the direction of $y$ axis without any rotation. Therefore, the stiffness constant of folded beam, $k_{d e}$, (in Fig.6.) under the work state is defined by the ratio of force, $F_{D}$, and deflection, $w_{D}, k_{d e}=F_{D} / w_{D}$.

The proof mass of accelerometer is supported by four folded beams arranged symmetrically and it can be modeled by a mass-spring system in Fig.7. The total stiffness constant of the mass-spring system is the sum of stiffness of four folded beams:

$$
k_{a}=k_{1}+k_{2}+k_{3}+k_{4}
$$

where $k_{i}$ is the stiffness constant of single folded beam. The values of constant are equal to each other under ideal condition, $k_{i}=k_{c}=6 E I_{z} / l^{3}$.[18] . The total stiffness changes when fabrication errors occur in one beam. The convex defect in this research leads one constant $k_{i}$ to another $k_{d e}$. The stiffness constant of accelerometer, therefore, becomes:

$$
k_{a c}=3 k_{c}+k_{d e}
$$

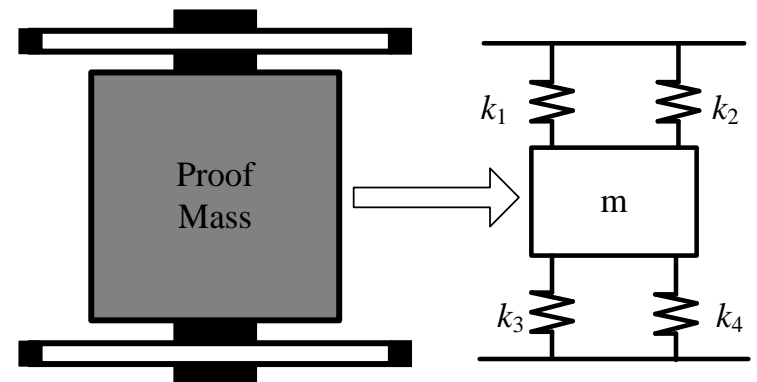

Fig.7. Accelerometer structure and its mechanical model.

It is concluded from the above analysis that the convex defect does not stop the proof mass from moving but does change the extent of its movability. The degree of change is dependent on the location of the convex defect which reversely can be identified by measuring the change of sensitivity of accelerometer.

\section{SimULATION \& OBSERVATION}

A model of the accelerometer having a folded beam with convex defect is developed by FEM software, as shown in Fig.8., and the dimension of single beam is $440 \mu \mathrm{m} \times 6 \mu \mathrm{m} \times 40 \mu \mathrm{m}$. The convex defect is anchored on beam $\mathrm{R}$ and its end has a small gap from beam $\mathrm{L}$. The convex is located at distance $a$ from the border of proof mass. The convex has the same thickness as beam while its width is $3 \mu \mathrm{m}$ and length is $12.99 \mu \mathrm{m}$.

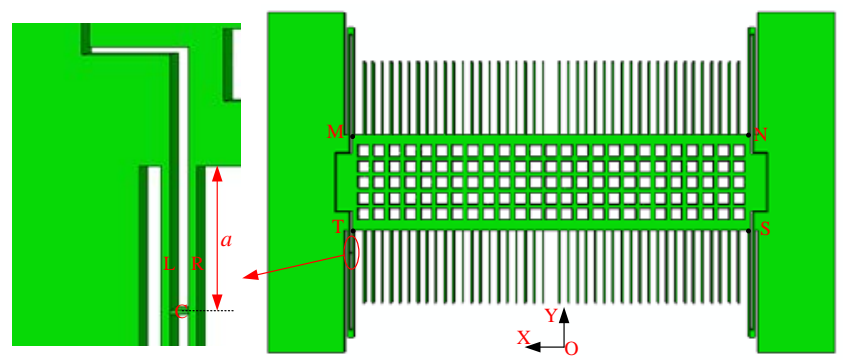

Fig.8. Full model of accelerometer with convex defect.

The position of the convex defect determines the level of stiffness change and varies from one accelerometer to another due to the uncertainty of micro fabrication. Fig.9. gives out the relationship of convex position and stiffness change. The digits on the horizontal axis represent the distance between the convex defect and border of proof mass while the digits on the vertical axis represent the ratio of the stiffnesses with defect and without defects, $r=k_{a} / k_{a c}$. The relative error is no more than $6.5 \%$, exhibiting a good 
agreement between analytical results and simulated ones. It shows that convex defect can cause a broad change of the accelerometer's stiffness in the sensitive direction.

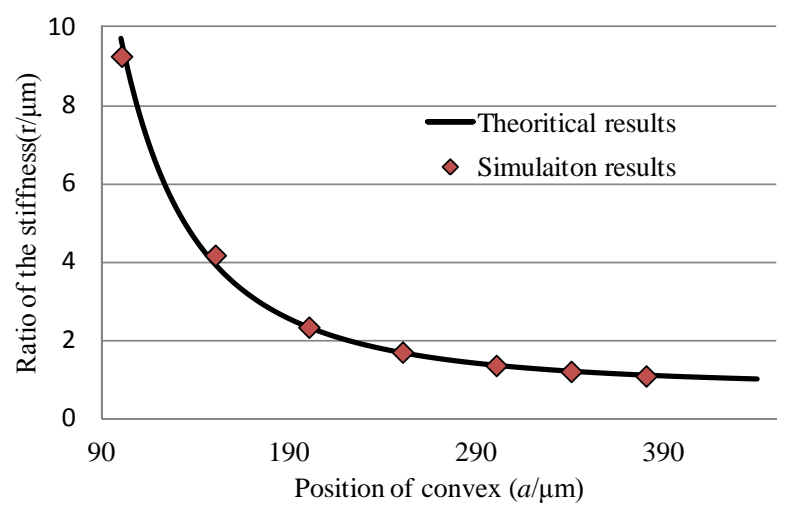

Fig.9. Influence of distance a on stiffness constant.

Although based on the assumption of taking the convex defect as rigid body during the analytical derivation, the results indicate that the analytical formula is accurate enough to access the influence of convex defect's location on the stiffness constant of accelerometers. Friction coefficient, for a more reasonable derivation, is also concerned. The displacements of points M, N, S, T of movable proof mass in Fig.8. is selected to study the effects of friction between convex and beam. Table 2. has compared the displacements of proof mass under the applied acceleration. The average displacements without friction of interested points are $20.3 \mathrm{~nm}$ under $5 \mathrm{~g}$. Compared with no friction, the friction with coefficient 0.1 and 0.2 only induces $0.079 \%$ and $0.151 \%$ decrease in the average displacements, respectively. The property of contacting surfaces, therefore, has little effects on the displacement.

Table 2. Displacements under different friction coefficients.

\begin{tabular}{ccccc}
\hline \multicolumn{2}{c}{ Friction coefficient } & 0 & 0.1 & 0.2 \\
\hline Displacement of & $\mathrm{M}$ & 20.305 & 20.292 & 20.275 \\
selected points & $\mathrm{N}$ & 20.303 & 20.286 & 20.272 \\
$(\mathrm{~nm})$ & $\mathrm{S}$ & 20.247 & 20.237 & 20.223 \\
& $\mathrm{~T}$ & 20.247 & 20.222 & 20.208 \\
\hline
\end{tabular}

The analytical derivation and FEM simulation show that the stiffness constant of folded beam exhibits a steep change when the contact between beam and convex occurs under bending deformation and its change scale depends on the location of convex defect on beams and has almost nothing to do with the contacting characteristics of interface. As for the studied accelerometer, its sensitivity jump fully results from the shift of beam stiffness. The value of sensitivity, according to (2), is reversely proportional to the total stiffness of folded beam. Hence, the sensitivity ratio can be represented by the stiffness ratio as:

$$
\frac{S_{a c}}{S_{a}}=\frac{1}{r}=\frac{k_{a c}}{k_{a}}
$$

where $S_{a c}$ is the changed sensitivity, $S_{a c}=103.5 \mathrm{mV} / g_{0} ; S_{a}$ is the original sensitivity, $S_{a}=166.9 \mathrm{mV} / g_{0}$.

The stiffness ratio induced by convex defects is $r=1.61$. The location of defect on the beam, based on Fig.9. and Reference [16], is $291.4 \mu \mathrm{m}$ from the border of proof mass block or is $148.6 \mu \mathrm{m}$ from the rigid joint. Fig.10. shows the results of measurement by microscopy compared with its perfect counterparts in SEM. The measured distance between convex defect and joint is about $150 \mu \mathrm{m}$ which has a great agreement with the theoretical prediction.

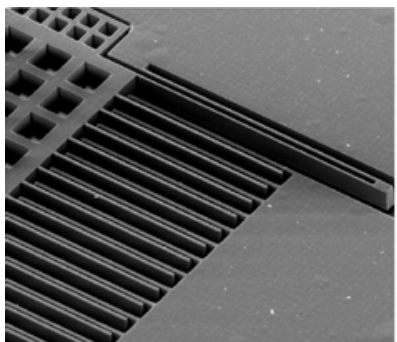

(a) SEM with perfect structure

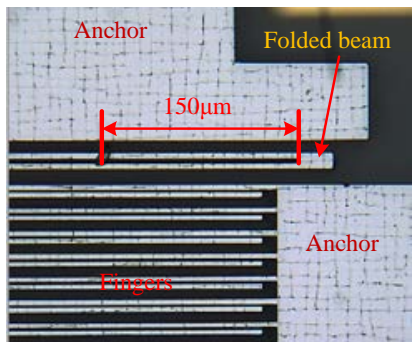

(b) Defected structure in microscopy
Fig.10. Measurement of defect location.

\section{CONCLUSION}

The sensitivity jump of a micro accelerometer is observed in the calibration which is an indispensable step before throwing accelerometers into markets. The stiffness variation of folded beam with convex defect is recognized as the only source of such an abnormal phenomenon. Both the analytical method and numerical method are utilized to investigate the impact of convex on the stiffness constant before and after the surface of beam contacts to the end of convex. Due to the small relative movement between two contacting surfaces and small effect of longitudinal force on the transverse deformation, the friction property is found to have almost no impact on the stiffness jump. The experiments and simulation both indicate that the proposed analytical prediction is effective to explain the sharp change of the output of accelerometer as the input acceleration increases, and the change occurs where the deformed beam starts to contact the convex defect.

\section{ACKNOWLEDGMENT}

This work was supported in part by NSAF (No. U1530132), National Natural Science Foundation of China (Nos.51505068, 51575090) and Fundamental Research Funds for the Central Universities (Grant No. ZYGX2015J085). The authors would like to acknowledge Shi Zhigui for their assistance in fabrication and experiments.

\section{REFERENCES}

[1] Frosio, I., Pedersini, F., Borghese, N.A. (2010). Formulation of stiffness constant and effective mass for a folded beam. Archives of Mechanics, 62, 405418. 
[2] Won, S.P., Golnaraghi, F. (2010). A triaxial accelerometer calibration method using a mathematical model. IEEE Transactions on Instrumentation and Measurement, 59, 2144-2153.

[3] Sipos, M., Paces, P., Rohac J. \& Novacek P. (2012). Analyses of Triaxial Accelerometer Calibration Algorithms. Sensors Journal, IEEE, 12, 1157-1165.

[4] Cobb, C.L., Agogino, A.M. (2010). Case-based reasoning for evolutionary MEMS design. Journal of Computing and Information Science in Engineering, 10, 031005.

[5] Ruzziconi, L., Ramini, A.H., Younis, M.I., Lenci, S. (2014). Theoretical prediction of experimental jump and pull-in dynamics in a MEMS sensor. Sensors, 14, 17089-17111.

[6] Ku, I.S.Y., Reddyhoff, T., Holmes, A.S., Spikes, H.A. (2011). Wear of silicon surfaces in MEMS. Wear, 271, 1050-1058.

[7] Meral, F.C., Basdogan, I. (2006). Design methodology for microelectromechanical systems. Case study: Torsional scanner mirror. Journal of Mechanical Design, 129, 1023-1030.

[8] Sardan, O., Alaca, B.E., Yalcinkaya, A.D., Bøggild, P., Tang, P.T., Hansen, O. (2007). Microgrippers: A case study for batch-compatible integration of MEMS with nanostructures. Nanotechnology, 18, 375501.

[9] Tanner, D.M., Parson, T.B., Corwin, A.D., Walraven, J.A., Wittwer, J.W., Boyce, B.L., Winzer, S.R. (2007) Science-based MEMS reliability methodology. Microelectronics Reliability, 47, 1806-1811.

[10] Iannacci, J. (2015). Reliability of MEMS: A perspective on failure mechanisms, improvement solutions and best practices at development level. Displays, 37, 62-71.

[11] He, X., Su, W., Peng, B., Zhou, W. (2012). Electrostatic compensation method in frequency robustness design of micro accelerometer. Journal of Micro/Nanolithography, MEMS, and MOEMS, 11, 043001.

[12] Zhou, W., Li, B., Peng, B., Su, W., He, X. (2012). Prediction of gap asymmetry in differential micro accelerometers. Sensors, 12, 6857-6868.

[13] Zhou, W., Yu, H., Peng, B., Shen, H., He, X., Su, W. (2013). Modeling the microstructure curvature of Boron-doped silicon in bulk micromachined accelerometer. Materials, 6, 244.
[14] Zhou, W., Chen, Y., Peng, B., Yang, H., Yu, H., Liu, H., He, X. (2014). Air damping analysis in comb microaccelerometer. Advances in Mechanical Engineering, 6.

[15] Zhou, W., Yu, H., Zeng, J., Peng, B., Zeng, Z., He, X., Liu, Y. (2015). Improving the dynamic performance of capacitive micro-accelerometer through electrical damping. Microsystem Technologies, 1-9.

[16] Senturia, S.D. (2001). Microsystem Design. Kluwer Academic Publishers.

[17] Yu, H., Zhou, W., Peng, B., He, X., Hao, X., Zeng, Z. (2014). Modeling the Boron-doping silicon beam by a multilayer model. Mathematical Problems in Engineering, 2014, 6.

[18] Frosio, I., Pedersini, F., Borghese, N.A. (2009). Autocalibration of MEMS accelerometers. IEEE Transactions on Instrumentation and Measurement, 58, 2034-2041.

[19] Wai, C.W., Azid, I.A., Majlis, B.Y. (2011). Theoretical analysis of stiffness constant and effective mass for a round-folded beam in MEMS accelerometer. Strojniski Vestnik / Journal of Mechanical Engineering, 57, 517-525.

[20] Bustillo, J.M., Howe, R.T., Muller, R.S. (1998). Surface micromachining for microelectromechanical systems. Proceedings of the IEEE, 86, 1552-1574.

[21] Chabloz, M., Sakai, Y., Matsuura, T., Tsutsumi, K. (2000). Improvement of sidewall roughness in deep silicon etching. Microsystem Technologies, 6, 86-89.

[22] McAuley, S.A., Ashraf, H., Atabo, L., Chambers, A., Hall, S., Hopkins, J., Nicholls, G. (2001). Silicon micromachining using a high-density plasma source. Journal of Physics D: Applied Physics, 34, 2769.

[23] Liu, H.C., Lin, Y.H., Hsu, W. (2003). Sidewall roughness control in advanced silicon etch process. Microsystem Technologies, 10, 29-34.

[24] Li, J., Liu, A.Q., Zhang, Q.X. (2006). Tolerance analysis for comb-drive actuator using DRIE fabrication. Sensors and Actuators A: Physical, 125, 494-503.

[25] Gere, J.M., Goodno, B.J. (2009). Mechanics of Materials. Cengage Learning.

Received January 03, 2016. Accepted August 10, 2016 